\title{
Erratum to: Smoothing combined generalized estimating equations in quantile partially linear additive models with longitudinal data
}

\author{
Jing Lv ${ }^{1}$ • Hu Yang' ${ }^{1}$ Chaohui Guo ${ }^{1}$
}

\section{Erratum to: Comput Stat DOI 10.1007/s00180-015-0612-8}

In the original publication of the article, the formula in Sect. 3.2 is incorrect. The correct version of the formula is given below:

$$
\begin{aligned}
\overline{\boldsymbol{\theta}}^{(k+1)}= & \overline{\boldsymbol{\theta}}^{(k)}+\left(\sum_{i=1}^{m} \boldsymbol{H}_{\boldsymbol{i}}{ }^{T} \boldsymbol{\Lambda}_{\boldsymbol{i}} \boldsymbol{V}_{\boldsymbol{i}}^{-1} \tilde{\boldsymbol{\Lambda}}_{i} \boldsymbol{H}_{\boldsymbol{i}}+\hat{\boldsymbol{G}}\right)^{-1} \\
& \times\left.\left(\sum_{i=1}^{m} \boldsymbol{H}_{\boldsymbol{i}}{ }^{T} \boldsymbol{\Lambda}_{\boldsymbol{i}} \boldsymbol{V}_{\boldsymbol{i}}^{-1} \tilde{\boldsymbol{S}}_{i}-\hat{\boldsymbol{G}} \boldsymbol{\theta}\right)\right|_{\boldsymbol{\theta}=\overline{\boldsymbol{\theta}}^{(k)}, \boldsymbol{\Omega}=\overline{\boldsymbol{\Omega}}^{(k)}} \\
\overline{\boldsymbol{\Omega}}^{(k+1)}= & \left\{\left(\sum_{i=1}^{m} \boldsymbol{H}_{i}{ }^{T} \boldsymbol{\Lambda}_{i} \boldsymbol{V}_{i}^{-1} \tilde{\boldsymbol{\Lambda}}_{i} \boldsymbol{H}_{i}+\hat{\boldsymbol{G}}\right)^{-1} \boldsymbol{\Gamma}_{n}(\boldsymbol{\theta}, \boldsymbol{\Omega})\right. \\
& \left.\times\left(\sum_{i=1}^{m} \boldsymbol{H}_{i}{ }^{T} \boldsymbol{\Lambda}_{i} \boldsymbol{V}_{i}^{-1} \tilde{\boldsymbol{\Lambda}}_{i} \boldsymbol{H}_{i}+\hat{\boldsymbol{G}}\right)^{-1}\right\}\left.\right|_{\boldsymbol{\theta}=\overline{\boldsymbol{\theta}}^{(k)}, \boldsymbol{\Omega}=\overline{\boldsymbol{\Omega}}^{(k)}}
\end{aligned}
$$

The online version of the original article can be found under doi:10.1007/s00180-015-0612-8.

$\otimes$ Chaohui Guo

guochaohui2010@126.com

1 College of Mathematics and Statistics, Chongqing University, Chongqing 401331, China 